\title{
Analysis of Self Efficacy and Social Physique Anxiety between women engaged in physical exercise and those having sedentary lifestyle
}

\author{
Meghna Basu Thakur ${ }^{1}$, Namrata Joshi ${ }^{2}$ \\ ${ }^{1}$ Assistant Professor and Head, Department of Psychology, R.D. National College, Bandra(W), Mumbai. \\ ${ }^{2}$ Assistant Professor (CHB), R.D. National College, Bandra (W), Mumbai. \\ E-mail-meghnabasuthakur@yahoo.com
}

\begin{abstract}
Background: A growing body of research suggests that factors like self-efficacy, self-esteem, anxiety, wellbeing are strongly associated with psychological health. Research on the importance of exercise in relation to the self -efficacy, anxiety related to body and exercise in women is scarce. The difference (if any) in the self-efficacy and other related variable like anxiety within the women who engage themselves by involving in physical exercise and those who aren't involved in any such exercise related activities, needs to be studies extensively in order to understand these constructs in relation to exercise and sedentary lifestyle.

Objective: The present study seeks to explore the in self-efficacy and social physical anxiety in women who are engaged in physical exercise and those who aren't involved in any such exercise related activities and having sedentary lifestyle.

Methods: The participants were women in the age range of 24-35 years who were engaged in physical exercise (gymnasium, aerobics class, dancing class, zumba) and women engaged in no physical activity pertaining any form or exercise (sedentary lifestyle). The participants in both the groups were given two scales to be completed with no time constraints. The two scales consisted of Exercise Self Efficacy Scale and The Social Physique Anxiety Scale. The data was analysed and the t test was calculated in order to identify if there are differences between the scores of two groups.

Results: The data collected from both the groups will be analyzed for descriptive statistics. The obtained mean score for Self efficacy for women involved with physical exercise was 33. On the other hand the obtained Mean Score for Self efficacy for women having sedentary lifestyle was 26.56. The obtained mean from the Social Physique Anxiety scores of the physical exercise group and sedentary lifestyle group was 25.9 and 38.73 respectively. $(p=0.0001)$. The further inferential analysis through $t$ test showed significant difference between the self-efficacy scores of both the groups. The self-efficacy scores were found to be higher in the group of participants who were involved in physical exercise as compared to those who had sedentary lifestyle. On the other hand, the social physique anxiety scores were found to be higher in the group of participants who reported having sedentary lifestyle as compared to the scores of the participants who reported engaging in exercise $(\mathrm{p}<0.05)$.

Conclusion: This study puts a light on how exercise can be related to mental health in terms of the psychological factors like self-efficacy and social physique exercise. The results found in this study do indicate that exercise and sedentary lifestyles are related to social physique anxiety and self- efficacy.
\end{abstract}

ABSTRACT

Keywords: Exercise, self-efficacy, Sedentary lifestyle, Social physical anxiety, women

(Paper received $-10^{\text {th }}$ March 2016, Peer review completed $-20^{\text {th }}$ April 2016, Accepted $-27^{\text {th }}$ April 2016) 


\section{INTRODUCTION}

Exercise is physical activity that is planned, structured, and repetitive for the purpose of conditioning any part of the body. Physical activity is defined as any bodily movement produced by skeletal muscles that result in energy expenditure. The energy expenditure can be measured in kilocalories. Physical activity in daily life can be categorized into occupational, sports, conditioning, household, or other activities. Exercise is a subset of physical activity that is planned, structured, and repetitive and has as a final or an intermediate objective the improvement or maintenance of physical fitness. Physical fitness is a set of attributes that are either health- or skill-related [1].

A sedentary lifestyle is a type of lifestyle with no or irregular physical activity. Sedentary behavior also refers to a group of behaviors that occur whilst sitting or lying down while awake and typically require very low energy expenditure. The low energy requirements distinguish sedentary behaviors from other behaviors that also occur whilst seated but require greater effort and energy expenditure, eg, using a rowing machine. Sedentary activities include sitting, reading, watching television, playing video games, and computer use for much of the day with little or no vigorous physical exercise. A sedentary lifestyle can contribute to many preventable causes of death. Screen time is the amount of time a person spends watching a screen such as a television, computer monitor, or mobile device. Excessive screen time is linked to negative health consequences.

Time spent in sedentary behaviors (e.g., standing or sitting and watching television) may reduce the amount of time available for physical activity [2]. For example, increased television watching for children has been linked to both decreased physical activity and higher obesity prevalence [3]. Adults who spent more time sitting or standing at work were half as likely to achieve regular moderate physical activity levels (outside of work) than those who spent time walking at work and two times less likely than those that did heavy labor [4].

Self-efficacy is the extent or strength of one's belief in one's own ability to complete tasks and reach goals. Self-efficacy affects every area of human endeavor. By determining the beliefs a person holds regarding his or her power to affect situations, it strongly influences both the power a person actually has to face challenges competently and the choices a person is most likely to make. These effects are particularly apparent, and compelling, with regard to behaviors affecting health. Self-efficacy has been found to be the most consistent psychosocial correlate of physical activity [5]. Self-efficacy is a situation-specific form of self-confidence [6] and has been frequently measured by having participants rate their confidence levels to overcome common barriers to physical activity [7]. Research has shown that participants who had a high level of confidence to complete physical activities, despite obstacles, were more likely to actually engage in exercise behaviors than participants with low exercise self-efficacy [8].

Hart, Leary, and Rejeski [9] defined the fear as social physique anxiety (SPA), or feelings of anxiety as a result of having one's physique evaluated by others. Social physique anxiety is a derivative of social anxiety directly related to the responses of the social judgment of one's physique. Physique was operationally defined by the work of Hart and colleagues [9] as "one's body form and structure, specifically body fat, muscle tone, and general body proportions." These anxious feelings are due to the social environment of exercise, thus creating anxiousness related to self-presentation and having one's body on display [10].

Exercise and physical activity have demonstrated a wide range of effects on a host of physical conditions including coronary artery disease, obesity, cancers, and overall mortality [11-12]. Within the domain of exercise, self-presentation has been studied predominantly in terms of social physique anxiety [13]. In line with self-presentation, social physique anxiety has been defined as an affective response that reflects concern about how one's body may be judged by others [14]. Although conceptually distinct, body image is related to the concept of social physique anxiety. In this respect, individuals may be concerned with how others view their physiques, either because their bodies are objectively unattractive or because they have an unrealistically negative perception of their physiques. Therefore, social physique anxiety is considered to be a type of social anxiety, defined by Hart et al. as the anxiety felt by people in response to others' perceived appraisals of their physique (the structure and shape of their bodies-height, weight, muscle tone). Social physique anxiety has been related to negative health-related behavioral and psychological 
consequences, such as low self-esteem and self-concept [15-16].; appearance and body image dissatisfaction [10]; smoking [17] and eating disorders [18]. Furthermore, most research on the relationship between exercise adherence and social physique anxiety has indicated a negative relationship between both variables [13]. In fact, due to their body image, adolescents may avoid or stop exercising, especially if they think that others might consider them fat or uncoordinated [17].

One factor that might influence the approach of those with high social physical anxiety towards disengagement of exercise could be the clothing worn in the exercise setting. Tight clothing is also often worn in exercise settings, thus potentially influencing those who are already insecure about their own physique to experience feelings of inferiority and stress [9]. Crawford and Eklund [10] conducted a study with college-aged females in which participants watched two video-taped aerobics classes. One tape included exercisers wearing t-shirts and shorts, which were not form-fitting, while the other tape included outfits which emphasized physique. It was found that college-aged females experienced higher levels of social physical anxiety (SPA) in settings with clothing that was form fitting and emphasized physique. Social physique anxiety levels have a tendency to influence physical self-perception as well. Researchers [19] conducted a three-year longitudinal analysis to determine if a relationship exists between the health behaviors (diet and activity level), emotions and perceptions (SPA and self-perception), and body composition (BMI) of adolescent females. Social Physical Anxiety (SPA) scores remained relatively consistent throughout the study, and the predictor variable most highly correlated with SPA was physical self-perception.

Russell [20] conducted a study assessing the prevalence of Social Physical Anxiety (SPA), self-esteem, and body image satisfaction among college-aged males. Results indicated that males scoring high in body dissatisfaction reported significantly higher SPA than those with low body dissatisfaction. Similar results were yielded by Russell and Cox [21] with a sample of college-aged females.

Another construct closely related to social physique anxiety is the relationship between SPA and selfefficacy. Albert Bandura [22] formulated a theory of self-efficacy, which states that people have a tendency to engage in activities that make them feel competent and increase self-confidence, including personal health habits [23]. Individuals may attempt to avoid situations causing anxiety due to uncertainty of skill competence or coping ability, whereas those situations where one feels efficacious decreased negative affect [22]. With heightened feelings of anxiety, those who have lower self-efficacy have a tendency to avoid certain exercise settings due to lack of perceived ability.

According to Bandura [23], the social-cognitive model of physical activity, self-efficacy is the primary determinant of consistent, health-promoting levels of physical activity. It is important to note, however, that the relationship between exercise beliefs and exercise behaviors is reciprocal. Behavior change is also determined by outcome expectations or sense of controllability; that is, whether one expects one's actions to lead to desirable outcomes. One may have high self-efficacy for exercise, but if one believes that exercise does not do anything to prevent or remediate aging-related losses, there would be little motivation to continue exercising [24].

Both self-efficacy and outcome expectations (sense of control) play an influential role in the adoption and maintenance of exercise behavior [25-26]. People who have higher levels of self-efficacy regarding exercise are both more likely to get involved with a program, and to realize that they are benefiting from its effects than are people low in self-efficacy [27]. While self-efficacy is believed to be an important component of physical activity initiation and maintenance, only a handful of interventions have sought to change efficacy beliefs among older adults [28].They reported that over the course of a 6-month exercise program, self-efficacy fluctuated throughout the intervention, and at program end, had declined. The authors suggested that this decline in self-efficacy may have been a result of the realization of the older adults that the program was coming to an end, and that they would have to exercise on their own. It is also possible that initial estimates of exercise efficacy were overly optimistic [28]. In another study, it was found out that perceived self-efficacy emerged as an important predictor of mental health among elderly males and females i.e. elderly who perceive themselves self-efficacious to have control over their environment reported better mental health and vice versa [29]. 


\section{METHODOLOGY}

The purpose of this study was to identify the effect on the Social Physical Anxiety as well as Self-efficacy amongst women who are engaged in physical exercise and those who are having sedentary lifestyle. The primary goal was to study the female population who tend to evaluate themselves more in terms of body image, ideal self-image, making them more prone to the negative self-evaluation and psychological disturbances. Hence, it is vital to study how there can be difference in the Social Physique Anxiety and Self-Efficacy within women with and without any involvement in physical exercise.

Hypothesis (Null Hypothesis): There is no difference in the Social Physique Anxiety scores obtained from women who are involved in physical exercise as compared to those women who reported having a sedentary lifestyle. There is no difference in the Self Efficacy scores obtained from women who are involved in physical exercise as compared to those women who reported having a sedentary lifestyle.

Alternate Hypothesis: There is a difference in the Social Physique Anxiety scores obtained from women who are involved in physical exercise as compared to those women who reported having a sedentary lifestyle. There is a difference in the Self efficacy scores obtained from women who are involved in physical exercise as compared to those women who reported having a sedentary lifestyle.

Independent Variable: The Independent Variable was presence and absence of physical exercise manipulated at two levels- women who were involved in physical exercise and women who had sedentary lifestyle with absence of any form of physical exercise in their daily routine. The physical exercise was defined as women who were engaged in any form of physical exercise by working out in gym, aerobics classes, yoga, different from of dancing (zumba, samba) from at least last six months for at least 30 minutes per day and for at least 5 days week. On the other hand sedentary lifestyle was defined as no involvement in any form of physical exercise with an intention to work out in physical form in daily routine.

Dependent Variable: The Dependent Variables were the total scores obtained by each participant in the following scales i.e. Exercise Self Efficacy Scale and The Social Physique Anxiety Scale (SPAS)

Design: Random groups design with one Independent Variable having two levels. The two groups consisted of the women in the age range of 24- 35 years. One group of participants (women) were those who were engaged in the physical exercise as described in the last segment $(n=30)$. While the other group of participants (women) were those who had sedentary lifestyle $(n=30)$ (no involvement in any form of physical exercise in daily routine).

Sample: The questionnaire was given to the women who were in the age range of 24-35 years old. One group consisted of women involved in any form of physical exercise by working out in gym, aerobics classes, yoga, different from of dancing (zumba, samba) from at least last six months for at least 30 minutes per day and for at least 5 days week. While the other group consisted of women who had sedentary lifestyle, where there was no form of physical exercise in which they were involved. This was analyzed by asking open ended questions about their daily routine. If the information given by the participant matched the research criteria essential for the groups, they were further asked to fill up the given questionnaires.

\section{Measures Used}

As described earlier, the participants had to first report if they were involved in any form of physical exercise in last six months for 30 minutes at least for 5 days a week. Based on this the experimental group of the participant was decided (Exercise group or sedentary lifestyle). Followed by which the research participants were given Exercise Self Efficacy Scale and The Social Physique Anxiety Scale (SPAS). The time to complete the self-report questionnaires was not restricted

The Exercise Self Efficacy Scale was used which is a self-report, pen and paper format measure. It measures perceived exercise self-efficacy for various types of physical activities. It requires individuals to indicate their confidence in performing physical activities and exercise. Exercise Self efficacy questionnaire 
had 10 items and requires individuals to respond to items on a 4-point likert scale (1-not at all true, 4always true). The summated score can be calculated where higher score indicating greater perceived Exercise Self Efficacy [30].

The Social Physique Anxiety Scale (SPAS) was developed by Hart, Leary, and Rejeski [9] and originally consisted of 12-items scored on a 5-point likert-type scale with anchors including 1 (not at all) to 5 (extremely). It consisted of statements concerning body physique or figure, which is body's form and structure; specifically, body fat, muscular tone, and general body proportions. The summated score can be calculated where higher score indicating greater perceived Social Physique Anxiety.

\section{STATISTICAL ANALYSIS}

Descriptive statistics including means and standard deviations were derived from the sample responses. The collected data was also analyzed for the inferential statistics in the form of t-test for random groups design with one independent variable having two levels was used. The $t$ tests were calculated using SPSS software (version 20). The mean scores obtained from both the groups (exercise group and sedentary lifestyle group) on Self efficacy Scale were compared with the help of $t$ test. The mean scores obtained from both the groups (exercise group and sedentary lifestyle group) on Rosenberg Self-Esteem Scale were compared through the help of $t$ test.

\section{RESULTS}

A total of 60 individuals participated in the research study. 30 participants were from the group where women reported being actively involved in physical exercise as per the standards set for the research criteria. On the other hand, 30 participants were women who had sedentary lifestyle with absence of any form of physical exercise in their daily routine. Both groups were well matched socio-demographically. The mean scores for social physique anxiety in the sedentary lifestyle group was $38.73 \pm 10.16$ while that in the exercise group was $25.9 \pm 8.39(\mathrm{p}<0.0001)$. The mean scores on exercise self efficacy in the sedentary lifestyle group was $33.0 \pm 4.94$ and in the exercise group was $26.57 \pm 6.92(p<0.0001)$. (Table 1). The obtained results have thus gathered evidence in support of the fact that there is a significant difference in the Exercise Self Efficacy (ESE) and social physique anxiety (SPA) scores obtained from women who are involved in physical exercise as compared to those women who reported having a sedentary lifestyle.

Table 1- Differences on the rating scales across both the groups

\begin{tabular}{|ccccc|}
\hline Rating Scale & $\begin{array}{c}\text { Exercise Group } \\
(\mathrm{n}=30)\end{array}$ & $\begin{array}{c}\text { Sedentary Group } \\
(\mathrm{n}=30)\end{array}$ & $\mathrm{t}$ value & $\mathrm{p}$ value \\
& \multicolumn{2}{c}{ Mean \pm SD } & & \\
\hline $\begin{array}{c}\text { Exercise Self } \\
\text { Efficacy Scale }\end{array}$ & $26.57 \pm 6.92$ & $33.0 \pm 4.94$ & $4.147(\mathrm{df}=58)$ & $<0.0001^{*}$ \\
\hline $\begin{array}{c}\text { Social Physique } \\
\text { Anxiety Scale }\end{array}$ & $25.9 \pm 8.39$ & $38.73 \pm 10.16$ & $5.336(\mathrm{df}=58)$ & $<0.0001^{*}$ \\
\hline
\end{tabular}

*significant (Independent sample $t$ test used in the assessment)

\section{DISCUSSION}

The primary goal was to study the female population who tend to evaluate themselves more in terms of body image, ideal self-image, making them more prone to the negative self-evaluation. Hence, it is vital to study how there can be difference in the self-esteem and self-compassion within women with and without any involvement to gym exercise. If physical activity can improve body composition outcomes, increase self-efficacy, and improve physical self-perceptions, it may represent the ideal construct to enhance intervention strategy in this population. However, surprisingly little research has been conducted 
examining the effects of physical activity on self-efficacy and social physique anxiety in middle-aged women. The present study provides evidence that social physique anxiety and self-efficacy are related to well-being in the exercise context, raising the possibility that the working on these constructs may help the individuals to actually focus on mental health, physical outcomes. Through the obtained results described in the previous section it is clear that both the hypothesis have got assembled support statistically. These results suggest much promise for the construct of social physique anxiety and self-efficacy as a way to promote a healthy conceptualization of the self for women exercisers.

In present society, it is a common occurrence for one's body to be on constant display. This can cause an array of emotions to arise as any social context is subject to human opinion and judgment. This can lead to social physique anxiety to be constantly at the higher end. The results obtained in the study also reflect the similar issue. Bandura [22] formulated a theory of self-efficacy, which states that people have a tendency to engage in activities that make them feel competent and increase self-confidence, including personal health habits [23]. Individuals may attempt to avoid situations causing anxiety due to uncertainty of skill competence or coping ability, whereas those situations where one feels efficacious decreased negative affect [22]. With heightened feelings of anxiety, those who have lower self-efficacy have a tendency to avoid certain exercise settings due to lack of perceived ability. This goes in line with the trend obtained in this study.

The group of women who were found to have low self -efficacy and high physical anxiety were those who weren't involved in any form of physical exercises. One of the possible reason to no involvement in physical activity might be due to the view that the task (physical exercise) is difficult which they may face challenging in mastering it. From the evidence obtained in this study it is clear that physical activity can be an effective intervention to enhance self-efficacy and elevate social physique anxiety. However, it is important to consider the underlying mechanisms for how and why physical activity improves self-efficacy and elevate social physique anxiety. By working on an intention to increase self-efficacy and elevate social physique anxiety of an individual, one would be more apt to engage in healthy behaviors leading to positive mental health- such as effectively dealing with stress, managing their weight, complying with a healthy diet, cessation of smoking, and adhering to exercise. It is important to note that for or many people it is difficult to start a regular exercise program because of numerous factors such as perceived barriers, lack of support, or low self-efficacy, financial support, motivation, accessibility to gym, awareness about the importance of physical exercise. Before generalizing the results it is crucial to consider these factors that can hamper one's intention to go for exercise regime.

The obtained results do give us an element that self-efficacy and anxiety are related to physical exercise. But, questions about whether self-efficacy influences motives to exercise and other exercise-related outcomes, whether it is self-efficacy that is impacted, or whether these relationships are bidirectional remain unanswered. It was ensured that the sample included women exercisers, which does limit the generalization of the findings limited to areas with similar population demographics. All data were selfreported thus the subjectivity in reporting the experiences was unavoidable. Further research is needed to compare the effect of varying exercise intensities and durations on both the constructs. Also more studies are necessary which examine the effects of different types of exercises, i.e. aerobic exercise vs. weight training, on self-esteem and self-efficacy. The potential for social desirability exists, as participants may alter survey or interview responses in efforts to make them feel more self-confident. Future studies should incorporate various physical indicators so that their impact on anxiety and efficacy can be determined.

\section{REFERENCES}

1. Caspersen CJ, Powell KE, Christenson GM. Physical activity, exercise, and physical fitness: definitions and distinctions for health-related research. Public Health Rep 1985;100(2):126-31.

2. Buckworth J, Nigg C. Physical activity, exercise, and sedentary behavior in college students. J Am Coll Health 2004;53(1):28-34.

3. Crespo CJ, Smit E, Troiano RP, Bartlett SJ, Macera CA, Andersen RE. Television watching, energy intake, and obesity in US children: results from the third National Health and Nutrition Examination Survey, 19881994. Arch Pediatr Adolesc Med 2001;155(3):360-5. 
4. Krüger S, Graf J, Merx MW, Stickel T, Kunz D, Koch KC, Hanrath P, Janssens U. The value of cardiopulmonary exercise testing and brain natriuretic peptide plasma levels in predicting the prognosis of patients with chronic heart failure. Eur J Intern Med 2006;17(2):96-101.

5. Trost SG, Pate RR, Sallis JF, Freedson PS, Taylor WC, Dowda M, Sirard J. Age and gender differences in objectively measured physical activity in youth. Med Sci Sports Exer 2002;34(2):350-5.

6. Cox RH. Sport psychology: Concepts and applications. McGraw-Hill; 1998.

7. Rhodes RE, Courneya KS, Blanchard CM, Plotnikoff RC. Prediction of leisure-time walking: an integration of social cognitive, perceived environmental, and personality factors. Int J Behav Nutr Phys Activity 2007;4(1):51-9.

8. Caspersen CJ, Christenson GM, Pollard RA. Status of the 1990 physical fitness and exercise objectives-evidence from NHIS 1985. Pub Health Rep 1986;101(6):587-92.

9. Hart EA, Leary MR, Rejeski WJ. The measurement of social physique anxiety. J Sport Ex Psychol 1989;11(1):94-104.

10. Eklund RC, Crawford S. Active women, social physique anxiety, and exercise. J Sport Ex Psychol 1994;16(4):431-48.

11. Blair SN, Clark DG, Cureton KJ, Powell KE. Exercise and fitness in childhood: implications for a lifetime of health. Persp Exer Sci Sports Med 1989;2:401-30.

12. Bouchard C, Shephard RJ, Stephens T, Sutton JR, McPherson BD. Exercise, fitness, and health: the consensus statement. In Exercise, fitness, and health: a consensus of current knowledge: proceedings of the International Conference on Exercise, fitness and health, May 29-June 3, 1988, Toronto, Canada.. 1990 (pp. 3-28). Human Kinetics Publishers ; 1990.

13. Hausenblas HA, Brewer BW, Van Raalte JL. Self-presentation and exercise. J Appl Sport Psychol 2004;16(1):3-18.

14. Leary MR. Self-presentational processes in exercise and sport. J Sport Ex Psychol 1992;14:339-51.

15. Gargari BP, Khadem-Haghighian M, Taklifi E, Hamed-Behzad M, Shahraki M. Eating attitudes, self-esteem and social physique anxiety among Iranian females who participate in fitness programs. J Sports Med Phys Fitness 2010;50(1):79-84.

16. Hagger MS, Wood CW, Stiff C, Chatzisarantis NL. Self-regulation and self-control in exercise: The strengthenergy model. Int Rev Sport Ex Psychol 2010;3(1):62-86.

17. Sabiston CM, McDonough MH, Crocker PR. Psychosocial experiences of breast cancer survivors involved in a dragon boat program: exploring links to positive psychological growth. J Sport Ex Psychol 2007;29(4):419-38.

18. Thompson AM, Chad KE. The relationship of social physique anxiety to risk for developing an eating disorder in young females. J Adolesc Health 2002;31(2):183-9.

19. Crocker PR, Eklund RC, Kowalski KC. Children's physical activity and physical self-perceptions. J Sports Sci 2000;18(6):383-94.

20. Cox RH, Martens MP, Russell WD. Measuring anxiety in athletics: The revised competitive state anxiety inventory-2. J Sport Ex Psychol 2003;25(4):519-33.

21. Russell WD, Cox RH. Social physique anxiety, body dissatisfaction, and self-esteem in college females of differing exercise frequency, perceived weight discrepancy, and race. J Sport Behav 2003;26(3):298-303.

22. Bandura A. Self-efficacy in changing societies. Cambridge university press; 1995.

23. Bandura A. Self-efficacy: The exercise of control. New York: Freeman ; 1997.

24. Lachman ME, Agrigoroaei S. Promoting functional health in midlife and old age: Long-term protective effects of control beliefs, social support, and physical exercise. PLoS one 2010;5(10):e13297.

25. Mirzaiinjmabadi K, Anderson D, Barnes M. The relationship between exercise, body mass index and menopausal symptoms in midlife Australian women. Int J Nurs Pract 2006;12(1):28-34.

26. Resnick B, Jenkins LS. Testing the reliability and validity of the self-efficacy for exercise scale. Nurs Res 2000;49(3):154-9.

27. Crocker PR, Eklund RC, Kowalski KC. Children's physical activity and physical self-perceptions. J Sports Sci 2000;18(6):383-94.

28. McAuley E, Jerome GJ, Marquez DX, Elavsky S, Blissmer B. Exercise self-efficacy in older adults: Social, affective, and behavioral influences. Ann Behav Med 2003;25(1):1-7.

29. Singh AP, Shukla A, Singh PA. Perceived self efficacy and mental health among the elderly. Delhi Psychiatry Journal 2010;13(2):314-21.

30. Shin Y, Jang H, Pender NJ. Psychometric evaluation of the exercise self-efficacy scale among Korean adults with chronic diseases. Res Nurs Health 2001;24(1):68-76.

Acknowledgements - Nil ; Source of Funding - Nil ; Conflict of Interest - Nil 